\title{
Analysis of recent type Ia supernova data based on evolving dark energy models
}

\author{
Jaehong Park, Chan-Gyung Park and Jai-chan Hwang \\ Department of Astronomy and Atmospheric Sciences, Kyungpook National University, Daegu, Korea
}

(Dated: October 7, 2018)

\begin{abstract}
We study characters of recent type Ia supernova (SNIa) data using evolving dark energy models with changing equation of state parameter $w$. We consider sudden-jump approximation of $w$ for some chosen redshift spans with double transitions, and constrain these models based on Markov Chain Monte Carlo (MCMC) method using the SNIa data (Constitution, Union, Union2) together with baryon acoustic oscillation $A$ parameter and cosmic microwave background shift parameter in a flat background. In the double-transition model the Constitution data shows deviation outside $1 \sigma$ from $\Lambda \mathrm{CDM}$ model at low $(z \lesssim 0.2)$ and middle $(0.2 \lesssim z \lesssim 0.4)$ redshift bins whereas no such deviations are noticeable in the Union and Union2 data. By analyzing the Union members in the Constitution set, however, we show that the same difference is actually due to different calibration of the same Union sample in the Constitution set, and is not due to new data added in the Constitution set. All detected deviations are within $2 \sigma$ from the $\Lambda$ CDM world model. From the $\Lambda$ CDM mock data analysis, we quantify biases in the dark energy equation of state parameters induced by insufficient data with inhomogeneous distribution of data points in the redshift space and distance modulus errors. We demonstrate that location of peak in the distribution of arithmetic means (computed from the MCMC chain for each mock data) behaves as an unbiased estimator for the average bias, which is valid even for non-symmetric likelihood distributions.
\end{abstract}

PACS numbers: 98.80.-k, 95.36.+x

\section{INTRODUCTION}

The type Ia supernova (SNIa) observation indicates current accelerated expansion of the universe [1, 2]. In order to explain the acceleration the concept of dark energy was introduced with many different realizations using a fluid, a field, etc. The SNIa data are important to study possible evolution of dark energy, often parametrized by an equation of state parameter $w=p / \rho c^{2} ; p$ is the pressure and $\rho c^{2}$ is the energy density of the dark energy component. Although the SNIa data have been continuously improving [3 8], it is still remarkable that all current observational data are well accommodated by the simple $\Lambda$ CDM model with $w=-1$. Thus, at the moment the observational data do not seriously demand evolution in the dark energy equation of state.

Although $\Lambda \mathrm{CDM}$ model explains observational data well, diverse time-varying dark energy models and analysis methods have been introduced to deal with increasing amount of data 10 41]. Possible evolution of dark energy has been probed mainly by Chevallier-PolarskiLinder (CPL) parametrization $w(a)=w_{0}+w_{a}\left(1-a / a_{0}\right)$ [40, 41] and the redshift-binned parametrization method based on the decorrelating technique [13, 22, 28]. In the latter, the equation of state parameter in each redshift bin is estimated independently of others based the piecewise constant, linear or cubic spline parametrizations. Compared with the CPL parametrization, which has only two free parameters with special evolution, the redshiftbinning method is more suitable to know specific dark energy variations because we can divide the redshift interval into many bins as we want. Although there is a restriction on the number of redshift bins due to insufficient data, the redshift-binning method is more appropriate to study the nature of dark energy.

There are several works showing tendency of $w$ deviating from -1 especially in the low redshift region 32 38]. The tendency indicates slow down of acceleration with the deceleration parameter $q$ increased in $z \leq 0.3$ [32]. Similarly, authors of Ref. 33] showed that $w$ is rapidly decreased as $z$ increases under both the CPL and the redshift-binned parametrization methods. In Ref. 34], the linear spline method was used to show that $w(z)$ can be oscillating around $w=-1$ with two turning points where $w<-1$ at $z \simeq 0.4$ and $w>-1$ at $z \simeq 1$. The authors of Ref. 35] also reported weak hint of possible evolution of dark energy; it is found that under the piecewise constant parametrization $w$ is less than -1 in $z$ between 0.25 and 0.75 . On the other hand, no significant evidence for dark energy evolution is found based on the similar methods in Refs. 36 38. In the high redshift region, authors of Ref. 39] report that the dark energy deviates from the cosmological constant at redshift $z \gtrsim 0.5$ based on analysis of SNIa and gamma-ray bursts (see also Ref. [34]).

In this paper we study the evolution of dark energy using a double sudden-jump transition model with varying transition redshift, which is a simple version of the piecewise constant parametrization method. To constrain our model we use the recent SNIa data such as Union [7], Constitution [8], Union2 9] samples, together with the baryon acoustic oscillation (BAO) parameter 42] and the cosmic microwave background (CMB) shift parameter [43 45]. Especially we investigate the characters of the recent SNIa data sets in view of the simple evolving dark energy models $(i)$ by comparing the results of the 
Union, the Constitution data sets, and the same Union members included in the Constitution sample and (ii) by analyzing the $\Lambda \mathrm{CDM}$ based mock data sets mimicking the Constitution and Union2 samples.

According to our double-transition model, we found that the Union sample and the same Union members in the Constitution sample, both of which are based on the same light-curve fit parameters, show different behaviors of dark energy equation of state due to different calibration processes applied to the distance moduli of the Union members. From the $\Lambda \mathrm{CDM}$ mock data analysis, we also found that inhomogeneous distribution of SNIa data points in the redshift space and inhomogeneous distance modulus errors cause biases in the dark energy equation of state parameters. We have demonstrated that the peak of the distribution of arithmetic means (estimated from thousands of mock data sets) can be used as the unbiased estimator for the average bias in the dark energy equation of state parameters.

The paper is organized as follows. We introduce our dark energy models in Sec. III and describe our analysis method and the observational data used in this study in Sec. [II] Our results are presented in Secs. IV and V] Section VI is a discussion of our analysis with conclusions.

\section{DARK ENERGY MODEL}

We consider a $w$ CDM model dominated by dark energy fluid with equation of state parameter $w$ and cold dark matter. For generally varying dark energy equation of state the Friedmann equation is given by

$$
\begin{aligned}
& E^{2}(z) \equiv \frac{H^{2}(z)}{H_{0}^{2}}=\Omega_{\mathrm{r} 0}(1+z)^{4}+\Omega_{\mathrm{m} 0}(1+z)^{3}+\Omega_{\mathrm{DE} 0} f(z)+\Omega_{K 0}(1+z)^{2} \\
& f(z) \equiv e^{3 \int_{0}^{z}[1+w(z)] d \ln (1+z)}
\end{aligned}
$$

where $w(z)$ is the dark energy equation of state parameter at redshift $z ; z \equiv a_{0} / a-1$ with $a(t)$ the cosmic expansion scale factor; $H \equiv \dot{a} / a$ is the Hubble parameter; a dot represents a derivative with respect to the cosmic time $t ; \Omega_{\mathrm{r}}, \Omega_{\mathrm{m}}$, and $\Omega_{\mathrm{DE}}$ are radiation, matter, and dark energy density parameters, respectively; $\Omega_{K}=-K /\left(a^{2} H^{2}\right)$; subscript 0 indicates present epoch. We set the present CMB temperature $T_{0}=2.725 \mathrm{~K}$ and the number of massless neutrino species $N_{\nu}=3.04$. In this work we consider dark energy models with piecewise constant dark energy equation of state, in which $w$ parameter is constant for given redshift bins with a sudden jump at transitions. As a simple case, we consider a double-transition model (with three redshift bins). By imposing conditions that $a$ and $\dot{a}$ are continuous at the transition epochs, we rewrite Eq. (2) as

$$
f(z)=\left\{\begin{array}{lc}
(1+z)^{3\left(1+w_{0}\right)} & z<z_{\operatorname{tr} 1} \\
(1+z)^{3\left(1+w_{1}\right)}\left(1+z_{\operatorname{tr} 1}\right)^{3\left(w_{0}-w_{1}\right)} & z_{\operatorname{tr} 1} \leq z<z_{\operatorname{tr} 2} \\
(1+z)^{3\left(1+w_{2}\right)}\left(1+z_{\operatorname{tr} 1}\right)^{3\left(w_{0}-w_{1}\right)}\left(1+z_{\operatorname{tr} 2}\right)^{3\left(w_{1}-w_{2}\right)} & z \geq z_{\operatorname{tr} 2}
\end{array}\right.
$$

where $w_{i}(i=0,1,2)$ is the dark energy equation of state parameter in each redshift bin; $z_{\operatorname{tr} 1}$ and $z_{\operatorname{tr} 2}$ represent the locations of transition. The general formula with arbitrary number of bins can be found in Refs. [28, 33]. We assume a flat background $\Omega_{K}=0$, thus $\Omega_{\mathrm{DE}}=1-\Omega_{\mathrm{r}}-\Omega_{\mathrm{m}}$. As we will consider models with fixed transition redshifts throughout this paper, we have five free parameters for our double-transition model. We denote the parameters as $\boldsymbol{\theta}=\left(h, \Omega_{\mathrm{m} 0}, w_{0}, w_{1}, w_{2}\right) ; h$ is a normalized present day Hubble parameter $H_{0} \equiv$ $100 h \mathrm{~km} \mathrm{~s}^{-1} \mathrm{Mpc}^{-1}$.

\section{METHOD AND DATA}

\section{A. Markov Chain Monte Carlo method}

To obtain likelihood distributions for parameters $\boldsymbol{\theta}$, we use the Markov Chain Monte Carlo (MCMC) method. The MCMC chain elements are generated to randomly explore the whole parameter space based on the Metropolis-Hastings algorithm [46, 47]. For the probability density function $P(\boldsymbol{\theta} \mid \mathbf{D})$ which is needed to make decisions for accepting/rejecting a randomly chosen chain 
element, we use

$$
P(\boldsymbol{\theta} \mid \mathbf{D}) \propto \exp \left(-\frac{\chi^{2}}{2}\right), \quad \chi^{2}=\sum_{i=1}^{N} \frac{\left[X_{i}(\boldsymbol{\theta})-X_{\mathrm{obs}, i}\right]^{2}}{\sigma_{\mathrm{obs}, i}^{2}},
$$

where $\mathbf{D}$ denotes data, $X_{i}(\boldsymbol{\theta})$ represents the model prediction for the $i$ th observed data point $X_{\mathrm{obs}, i}$ with measurement error $\sigma_{\mathrm{obs}, i}$, and $N$ is the total number of data points. We use SNIa, BAO, and CMB data to constrain our model. In this case the final $\chi^{2}$ is the sum of individual $\chi^{2}$ 's: $\chi^{2}=\chi_{\mathrm{SN}}^{2}+\chi_{\mathrm{BAO}}^{2}+\chi_{\mathrm{CMB}}^{2}$.

In applying the MCMC method to our dark energy models, we impose two priors. First, we impose a prior that the dark energy at sufficiently early epoch $\left(z_{i}=10^{8}\right)$ should be lower than the maximum amount allowed by the big bang nucleosynthesis calculation, $\Omega_{\mathrm{DE} i}<0.045$ [48]. If the number and the quality of SNIa data points within a redshift bin are not sufficient, the MCMC chain of the dark energy equation of state parameter of the bin usually does not converge, gradually decreasing to smaller and smaller values, sometimes reaching -100 in an extreme case. Therefore, as the second prior we set lower bounds to all the dark energy equation of state parameters, $w_{i}>-5$, to make sure the convergence of the MCMC chain.

In applying the MCMC method to the observational data, we generate one million chain elements after a burning period during which the first two thousand chain elements are discarded. For a $\Lambda$ CDM based mock data set (see Sec. IIIC below), we generate 0.2 million chain elements. To test the convergence of MCMC chains we use a simple diagnostic: the means calculated from the first (after burning process) and the last $10 \%$ of the chain becomes approximately equal to each other if the chain has converged (see Appendix B of Ref. [49] for detailed description). We found that the 0.2 million chain elements are sufficient for the convergence of our MCMC chains. For each dark energy equation of state parameter in our models, we obtain its one-dimensional likelihood distribution by marginalizing over all other parameters, and compute the one-dimensional likelihood peak value or the arithmetic mean value as our average estimate, together with $1 \sigma(68.3 \%)$ and $2 \sigma(95.4 \%)$ confidence limits.

It should be noted that in our simple piecewise constant parametrization of dark energy equation of state we do not apply the decorrelating technique to obtain the independent estimate of $w_{i}$ 's. Instead, by comparing the $w_{i}$ 's measured from the observational data with the likelihood confidence regions that the $\Lambda$ CDM mock data analysis allows, we obtain a desirable statistical criterion for whether the measured deviation can be interpreted as dark energy evolution or not.

\section{B. Observational data}

To constrain our dark energy model we jointly use the three kinds of observational data such as the SNIa data,
BAO $A$, and CMB $R$ parameters.

For the SNIa data, we use the Union sample (307 members) [7], the Constitution sample (397 members) [8], and the Union2 sample (557 members) [9]. For Union and Union2 data analysis, we use the covariance matrix between distance modulus errors without systematics. The Constitution data set consists of the same SNIa members in the Union data set and 90 CfA3 SNIa added at low redshift $(z<0.1)$. We will analyze four data sets: the Constitution set, the Union set, pure Union sample present in the Constitution set (hereafter Constitution$\mathrm{U})$, and Union2 set. The SNIa distance modulus in the Constitution- $\mathrm{U}$ set is different from the one in the original Union set due to a new calibration used in the Constitution data set.

Authors of Ref. 8] produced the Constitution data set by taking directly the SALT light-curve fit parameters of the Union set (listed in Table 11 of Ref. [7]) and adding them to the SALT output of CfA3 sample. They found the best-fit cosmology from the combined SALT output parameters (rest-frame peak magnitude $m_{B}$, stretch $s$, color $c$ ) by using the empirical relation for the distance modulus

$$
\mu=m_{B}-M_{B}+\alpha(s-1)-\beta c
$$

obtaining empirical coefficients related to stretch correction $\left(\alpha=1.34_{-0.08}^{+0.08}\right)$, color correction $\left(\beta=2.59_{-0.08}^{+0.12}\right)$, and absolute magnitude $\left(M_{B}\right)$, which are different from those used in the Union data analysis $(\alpha=1.24 \pm 0.10$, $\beta=2.28 \pm 0.11 ;$ [7]). Furthermore, in the Constitution data set, a host galaxy peculiar velocity of $400 \mathrm{~km} \mathrm{~s}^{-1}$ has been assumed (rather than $300 \mathrm{~km} \mathrm{~s}^{-1}$ used in the Union data set [7]), and the distance modulus uncertainties of Union SNIa members have been modified to reproduce the same amount of uncertainty in the measurement of $w$ (based on the constant $w$ dark energy model) as in Ref. 7]. Besides, an uncertainty of 0.138 mag has been added in quadrature to the uncertainties of SALT light-curve fit for each CfA3 SNIa. Since different empirical coefficients and different level of additional uncertainties were used to calculate the distance modulus of each SNIa, the Union and the Constitution sets have experienced different calibration process (see Ref. [8] for a complete description). Figure1 compares distance moduli of Constitution-U and Union SNIa samples, where for each SNIa member the mean-subtracted difference between two distance moduli divided by the measurement uncertainty is shown. Although distance modulus values from two samples do not show special trend, the plot shows a scatter in both positive and negative directions, implying that SNIa members of Constitution- $U$ and Union samples experienced the different calibration process.

For a given background world model the distance modulus at redshift $z$ is given by

$$
\mu \equiv m-M=5 \log _{10}\left[\frac{(1+z) r(z)}{1 \mathrm{Mpc}}\right]+25
$$




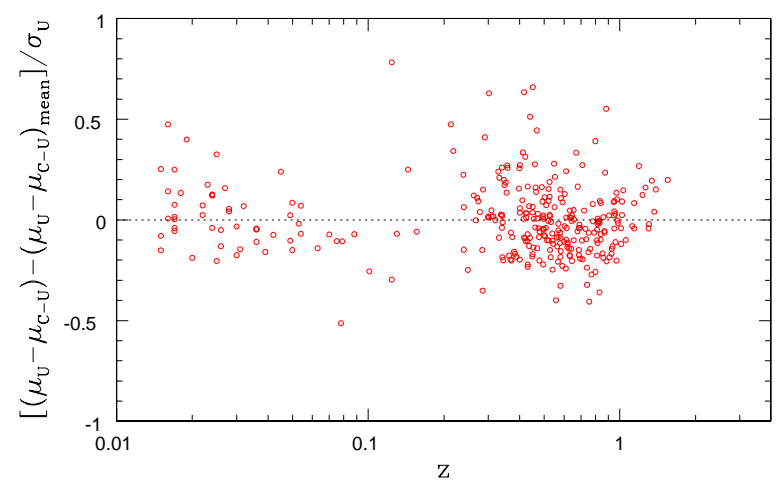

FIG. 1: A plot showing the difference between Constitution$\mathrm{U}\left(\mu_{\mathrm{C}-\mathrm{U}}\right)$ and Union $\left(\mu_{\mathrm{U}}\right)$ SNIa distance modulus relative to the measurement error. Since each data set has an arbitrary zero point in the distance modulus, actually shown are meansubtracted differences between two distance moduli divided by the distance modulus uncertainty of the Union SNIa $\left(\sigma_{\mathrm{U}}\right)$.

with the comoving distance at redshift $z$ given as

$$
r(z)=\frac{c}{H_{0} \sqrt{\Omega_{K 0}}} \sin \left[\sqrt{\Omega_{K 0}} \int_{0}^{z} \frac{H_{0}}{H(z)} d z\right] .
$$

The SNIa data provide a distance modulus $\mu_{\mathrm{obs}, i}$ with a measurement error $\sigma_{\mathrm{obs}, i}$ for each SNIa at redshift $z_{i}$. We obtain $\chi^{2}$ for the SNIa data as

$$
\chi_{\mathrm{SN}}^{2}=\sum_{i=1}^{N_{\mathrm{SN}}} \frac{\left[\mu_{i}(\boldsymbol{\theta})-\mu_{\mathrm{obs}, i}\right]^{2}}{\sigma_{\mathrm{obs}, i}^{2}},
$$

where $N_{\mathrm{SN}}$ is the number of SNIa in the data set, and $\mu_{i}(\boldsymbol{\theta})$ is the distance modulus at redshift $z_{i}$ predicted by the theoretical world model to be constrained.

The BAO A parameter is defined as [42]

$$
A \equiv \Omega_{\mathrm{m} 0}^{1 / 2} E\left(z_{b}\right)^{-1 / 3}\left[\frac{1}{z_{b}} \int_{0}^{z_{b}} \frac{d z^{\prime}}{E\left(z^{\prime}\right)}\right]^{2 / 3} .
$$

From the Sloan Digital Sky Survey luminous red galaxy sample we have [4]

$$
z_{b}=0.35, \quad A_{\mathrm{obs}}=0.469\left(\frac{n_{s}}{0.98}\right)^{-0.35} \pm 0.017,
$$

where $n_{s}$ is the spectral index of scalar-type perturbation. We take $n_{s}=0.960$ based on the Wilkinson Microwave Anisotropy Probe (WMAP) 5-year results [43]. The $\chi^{2}$ of $\mathrm{BAO}$ data is

$$
\chi_{\mathrm{BAO}}^{2}=\frac{\left[A(\boldsymbol{\theta})-A_{\mathrm{obs}}\right]^{2}}{\sigma_{\mathrm{obs}, A}^{2}},
$$

with $\sigma_{\mathrm{obs}, A}=0.017$ [42].

We use CMB shift parameter $R$ introduced as [44, 45]

$$
R \equiv \sqrt{\Omega_{\mathrm{m} 0} H_{0}^{2} / c^{2}} \cdot r\left(z_{*}\right),
$$

where $z_{*}$ is the redshift of recombination. From the WMAP 5-year data we have [43]

$$
z_{*}=1090, \quad R_{\mathrm{obs}}=1.710 \pm 0.019 .
$$

The $\chi^{2}$ of the $R$ parameter is

$$
\chi_{\mathrm{CMB}}^{2}=\frac{\left[R(\boldsymbol{\theta})-R_{\mathrm{obs}}\right]^{2}}{\sigma_{\mathrm{obs}, R}^{2}},
$$

with $\sigma_{\mathrm{obs}, R}=0.019$ [43].

\section{Mock data}

In our estimation of $w_{i}$ at each redshift bin, the deviation from $\Lambda \mathrm{CDM}$ value can be interpreted as the evolution of dark energy. However, even the observational data obtained in a perfect $\Lambda \mathrm{CDM}$ world may give a spurious deviation from $w=-1$ due to inhomogeneous distribution of data points in the redshift space and the measurement errors, which makes the detection of dark energy evolution more complicated. To include such a spurious statistical effect in our analysis, we have generated thousands of $\Lambda$ CDM-motivated mock data sets and analyzed them in a similar way as the real observational data is analyzed.

For SNIa mock data, we make the five thousand Constitution and Union2 mock data sets predicted in the $\Lambda \mathrm{CDM}$ model by using redshifts $\left(z_{i}\right.$ 's) and distance modulus measurement errors $\left(\sigma_{\mathrm{obs}, i}\right.$ 's $)$ of SNIa members in the Constitution and the Union2 samples. For $\Lambda \mathrm{CDM}$ background world model we use cosmological parameters that are consistent with the WMAP 5-year observation, i.e., $h=0.705, \Omega_{\mathrm{m} 0}=0.274, \Omega_{K 0}=0$, and $w=-1$ [43]. For a given SNIa at redshift $z_{i}$, the mock data point is generated by $\mu_{\text {mock }, i}=\mu_{\Lambda \mathrm{CDM}, i}+e_{i}$, where $\mu_{\Lambda \mathrm{CDM}, i}$ is a distance modulus predicted in the $\Lambda \mathrm{CDM}$ model and $e_{i}$ is a random number drawn from a Gaussian normal distribution with variance $\sigma_{\mathrm{obs}, i}^{2}$, which mimics the measurement noise. In this manner, we can get $\Lambda \mathrm{CDM}$ based SNIa mock data sets with the same redshift distribution and distance modulus precision of Constitution and Union2 samples. The same method is applied to generating mock BAO $A$ and CMB $R$ parameters. The similar technique of generating SNIa mock data sets has been used in Refs. [50, 51].

There are two good points of using $\Lambda \mathrm{CDM}$ mock data sets. First, we can include the statistical effect induced by different random realizations of measurement errors, which usually appears as additional variance in our estimation of dark energy equation of state parameters. Thus, the confidence regions of $w_{i}$ 's obtained from the $\Lambda \mathrm{CDM}$ mock data sets become bigger than those from the single data set (see Figs. 3 and 4 below). By comparing results from the observational data set with those from $\Lambda$ CDM mock data sets, we can avoid a false signature of dark energy evolution due to statistically peculiar measurement noise in the observational data. Analyzing 
the single data set cannot account for such a statistical effect. Second, we can estimate any bias in the estimate of $w_{i}$ relative to -1 , induced by the sparse number of data points and measurement errors within the redshift bin considered. We investigate this issue in detail in Sec. V

\section{CONSTRAINTS ON DARK ENERGY EQUATION OF STATE IN DOUBLE-TRANSITION MODELS}

In this section, we present the results of our dark energy model with double transitions. Figure 2 shows $w_{0}$, $w_{1}$, and $w_{2}$ measured from Constitution, ConstitutionU, Union, and Union2 SNIa samples together with BAO and $\mathrm{CMB}$ data, for various transition redshifts. We fix the width between the two transitions as $z_{\operatorname{tr} 2}-z_{\operatorname{tr} 1}=0.2$, and vary $z_{\operatorname{tr} 1}$ from 0.1 to 0.5 with steps of 0.1 . From the marginalized one-dimensional likelihood distributions of $w_{0}, w_{1}$, and $w_{2}$ parameters, we have estimated peak locations (denoted as P) and $1 \sigma(68.3 \%), 2 \sigma(95.4 \%)$ confidence limits (points with error bars). In the Constitution and Constitution- $\mathrm{U}$ sets $w_{0}$ is larger than -1 and $w_{1}$ is smaller than -1 for $z_{\operatorname{tr} 1} \leq 0.4$. However, such trends are not seen in the Union and Union2 sets. Values of $w_{2}$ tend to be larger than -1 in all four data sets. Since the Constitution- $\mathrm{U}$ sample is composed of exactly the same SNIa members of the Union data set, we can conclude that such different behaviors of $w_{0}$ and $w_{1}$ arise not due to the new CfA3 data added in the Constitution set but due to the different calibration applied to each data set.

Now, we consider a case of $z_{\mathrm{tr} 1}=0.2$ and $z_{\mathrm{tr} 2}=0.4$ where the deviations in Constitution and Constitution$\mathrm{U}$ sets are most significant. The result is shown in the top row of Fig. 3. In the Constitution and Constitution$\mathrm{U}$ sets the $1 \sigma$ confidence limits of all $w_{i}$ 's deviate from $w=-1$. However, in the cases of Union and Union2, $w_{0}$ and $w_{1}$ are consistent with $\Lambda$ CDM whereas $1 \sigma$ limits of $w_{2}$ deviate from $w=-1$. In order to estimate the statistical significance of the trends, we have also analyzed $\Lambda$ CDM based Constitution mock data sets, and estimated $68.3 \%$ and $95.4 \%$ confidence limits on $w_{i}$ 's based on the total sum of five thousand MCMC chains; these are shown as grey regions in Fig. 3 (top panels). The likelihood confidence regions for the $\Lambda$ CDM mock data sets are wider than those determined from the marginalized one-dimensional likelihood distributions for the Constitution data set (red error bars). This is because the mock data sets include the additional statistical variance caused by different realizations of measurement errors.

As a second measure of average, we compute the arithmetic mean (denoted as M) of the MCMC chain elements for each cosmological parameter. In fact, it is known that in the MCMC method the arithmetic mean of MCMC chain is appropriate as the best estimate for the parameter and is a more robust quantity than the peak (or mode) of the marginalized distribution [52]. The bot- tom row of Fig. 3 shows distributions of five thousand $M$ values of $w_{0}, w_{1}$, and $w_{2}$, each estimated from the MCMC chain of individual mock data set. The smooth distributions (shown as dark red curves) have been generated by applying the Savitzky-Golay smoothing filter 53. to the histograms with the bin size $\Delta w=0.02$. The distribution for $w_{1}$ is wider than those for $w_{0}$ and $w_{2}$, implying that the equation of state parameter $w_{1}$ is less tightly constrained in our double-transition model with $z_{\text {tr1 }}=0.2$; this is due to different number of data in each bin. For a comparison, M values for the observed SNIa data sets are indicated by vertical lines. In Constitution and Constitution- $\mathrm{U}$ sets all $\mathrm{M}$ values of $w_{i}$ 's are outside $1 \sigma$ regions of the distributions. In the Union set, the $\mathrm{M}$ values of $w_{0}$ and $w_{1}$ are consistent with the $\Lambda \mathrm{CDM}$ mock data, whereas that of $w_{2}$ is outside the $1 \sigma$ region. Although we detect more than $1 \sigma$ deviations of all $w_{i}$ 's in the Constitution and the Constitution- $U$ sets, we would like to emphasize that all the deviations are still within $2 \sigma$ regions, consistent with $\Lambda$ CDM model. The similarity between Constitution and Constitution-U sets and the difference between Constitution-U and Union are important because the Constitution- $U$ and the Union sets are based on the same SALT light-curve fit parameters of the Union SNIa sample. The different behaviors of $w_{0}$ and $w_{1}$ between the Constitution- $\mathrm{U}$ and the Union sets are purely due to different calibration processes applied in producing the SNIa distance modulus (see Sec. IIIB).

Table I compares equation of state parameters and matter density parameter in the double-transition dark energy model with $z_{\operatorname{tr} 1}=0.2$ and $z_{\operatorname{tr} 2}=0.4$ for various SNIa data sets and average estimation methods. For $\Lambda$ CDM based Constitution mock data sets, first we obtain distributions of $\mathrm{P}$ or $\mathrm{M}$ values and then present the peak of $\mathrm{P}$ distribution ( $\mathrm{PP}$ ), the peak of $\mathrm{M}$ distribution (PM), and the mean of $\mathrm{M}$ distribution (MM), together with $68.3 \%$ confidence limits. The location of peak and the confidence limits are determined based on the smooth distribution generated by applying the Savitzky-Golay smoothing filter to the histograms of $\mathrm{P}$ or $\mathrm{M}$ values. Comparison of the parameters estimated by three different average methods (PP, PM, and MM) with the fiducial value $w=-1$ suggests that $w_{1}$ is biased to the positive direction by the amount of about $0.07-$ 0.09. The estimated bias is uncertain roughly within $\sigma / \sqrt{N} \simeq 0.45 / \sqrt{5000}=0.006$. The $w_{0}$ is very weakly biased to the negative direction. For $w_{2}$ parameter, the presence of bias is not clear at this point because the three average estimates PP, PM, and MM indicate positive, zero-consistent, and negative biases relative to $w=-1$, respectively.

We notice all the observational data sets show a trend that $\mathrm{P}$ value is larger than $\mathrm{M}$ value for $w_{2}$ parameter, $w_{2}(\mathrm{P})>w_{2}(\mathrm{M})$ unlike other parameters. The similar feature is seen in the results of Constitution mock data analysis (PP and PM values in Table I). As shown in bottom row of Fig. 3, in the case of Constitution mock data sets $\mathrm{M}$ distributions for $w_{0}$ and $w_{1}$ look symmetric 

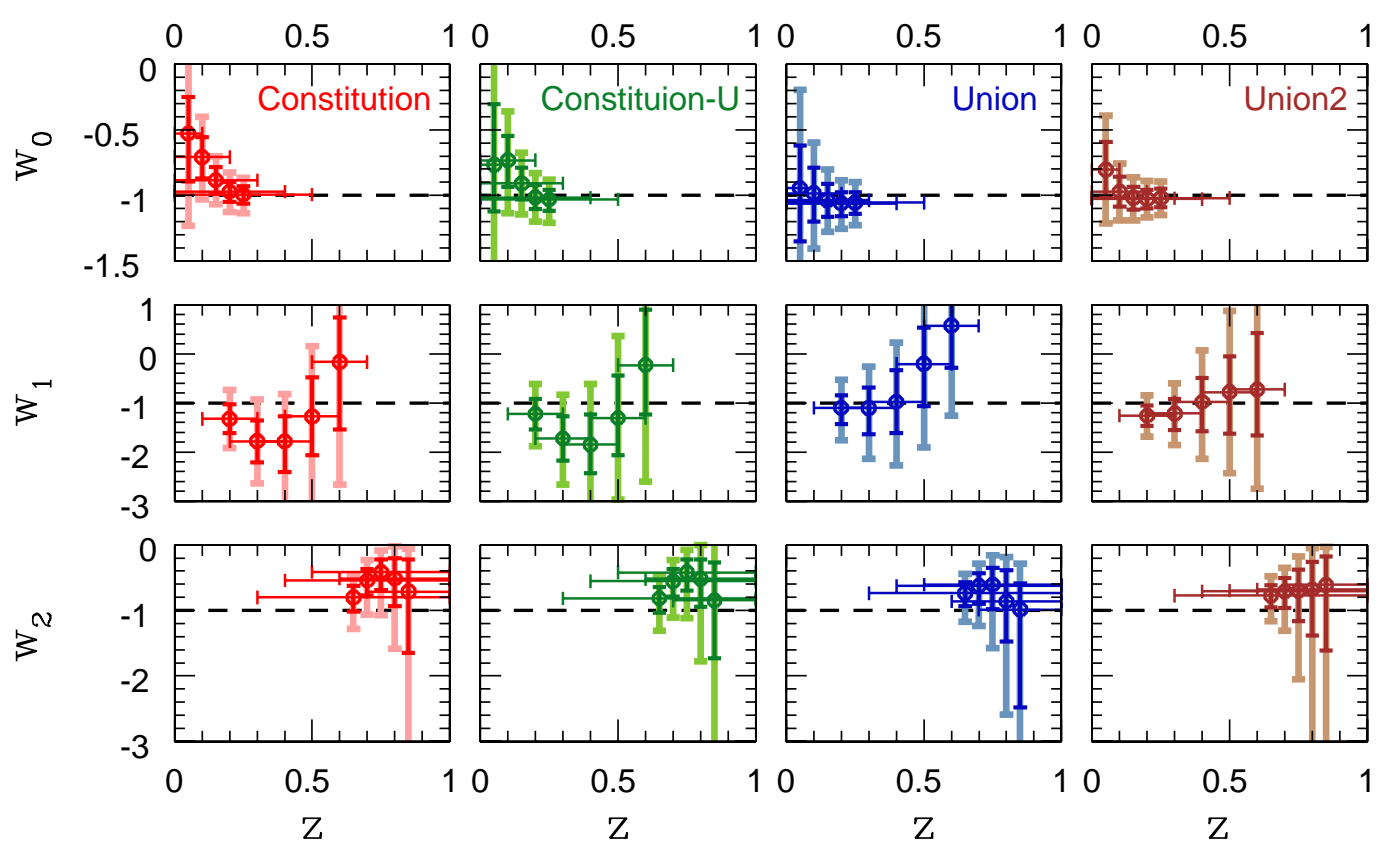

FIG. 2: Estimated $w_{0}, w_{1}$, and $w_{2}$ in the double-transition dark energy models. The transition redshift $z_{\mathrm{tr} 1}$ is varied from 0.1 to 0.5 by 0.1 increase in redshift while the width between the two transitions is fixed as $z_{\operatorname{tr} 2}-z_{\operatorname{tr} 1}=0.2$. Red, green, blue, and brown colors represent Constitution, Constitution-U, Union, and Union2, respectively. The error bars with dark (light) color indicate $68.3 \%(94.5 \%)$ confidence limits.

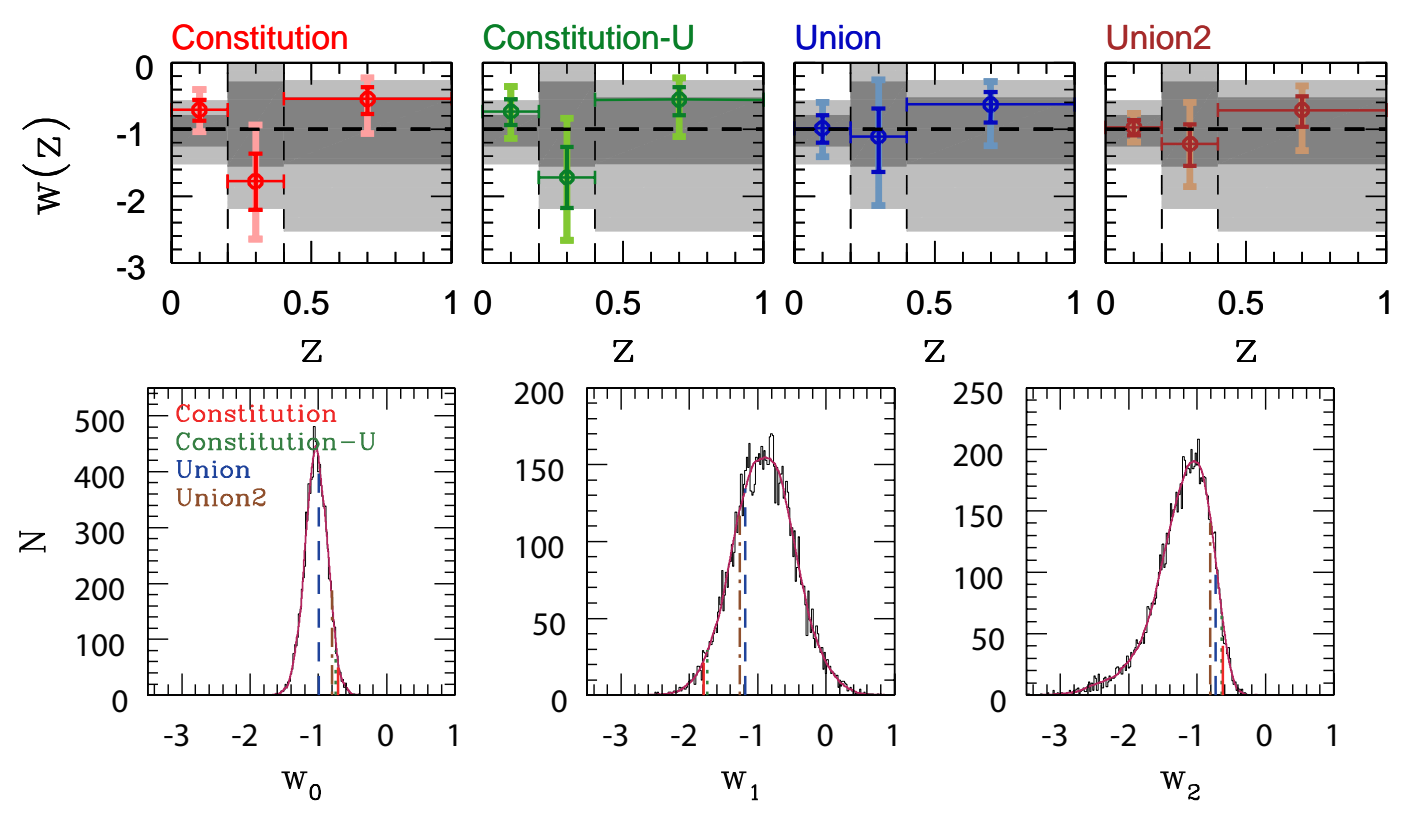

FIG. 3: Top panels: Estimated $w_{0}, w_{1}$, and $w_{2}$ in the double-transition dark energy model with $z_{\operatorname{tr} 1}=0.2$ and $z_{\operatorname{tr} 2}=0.4$, taken from Fig. 2 with the same colored code. Data points with error bars are based on the actual SNIa data sets. In all panels, shaded regions indicating $68.3 \%$ (dark grey) and $95.4 \%$ (light grey) confidence limits have been obtained from the five thousand $\Lambda$ CDM based Constitution mock data sets. Bottom panels: Distributions of arithmetic mean (M) values of $w_{0}, w_{1}$, and $w_{2}$ obtained from the Constitution mock data sets (histograms fitted by dark red smooth curves, see text). The three bins in the Constitution set have 147, 49, and 201 SNIa data points, respectively. Red (solid), green (dotted), blue (dashed), and brown (dot-dashed) vertical lines denote locations of $\mathrm{M}$ values for Constitution, Constitution-U, Union, and Union2 data sets, respectively. 
TABLE I: Estimated $w_{0}, w_{1}, w_{2}$, and $\Omega_{\mathrm{m} 0}$ in the double-transition dark energy model for $z_{\operatorname{tr} 1}=0.2$ and $z_{\operatorname{tr} 2}=0.4$. For comparison, results from the five thousand $\Lambda$ CDM based Constitution mock data sets are presented in the bottom. Symbols in the parenthesis represent our methods of average estimation, and the errors indicate $68.3 \%$ confidence limits (see text).

\begin{tabular}{cccccc}
\hline Data & Average & $w_{0}$ & $w_{1}$ & $w_{2}$ & $\Omega_{\mathrm{m} 0}$ \\
\hline Constitution & $(\mathrm{P})$ & $-0.71_{-0.16}^{+0.15}$ & $-1.78_{-0.43}^{+0.42}$ & $-0.54_{-0.23}^{+0.18}$ & $0.292_{-0.018}^{+0.017}$ \\
Constitution-U & $(\mathrm{P})$ & $-0.73_{-0.20}^{+0.19}$ & $-1.73_{-0.45}^{+0.45}$ & $-0.55_{-0.23}^{+0.19}$ & $0.291_{-0.019}^{+0.019}$ \\
Union & $(\mathrm{P})$ & $-0.98_{-0.22}^{+0.20}$ & $-1.11_{-0.54}^{+0.42}$ & $-0.63_{-0.27}^{+0.19}$ & $0.283_{-0.018}^{+0.017}$ \\
Union2 & $(\mathrm{P})$ & $-0.97_{-0.12}^{+0.11}$ & $-1.22_{-0.33}^{+0.30}$ & $-0.71_{-0.25}^{+0.21}$ & $0.278_{-0.017}^{+0.014}$ \\
\hline Constitution & $(\mathrm{M})$ & $-0.71_{-0.16}^{+0.15}$ & $-1.79_{-0.42}^{+0.43}$ & $-0.62_{-0.15}^{+0.26}$ & $0.294_{-0.016}^{+0.015}$ \\
Constitution-U & $(\mathrm{M})$ & $-0.74_{-0.19}^{+0.18}$ & $-1.74_{-0.44}^{+0.46}$ & $-0.64_{-0.13}^{+0.28}$ & $0.292_{-0.018}^{+0.017}$ \\
Union & $(\mathrm{M})$ & $-1.00_{-0.20}^{+0.22}$ & $-1.18_{-0.47}^{+0.49}$ & $-0.73_{-0.17}^{+0.29}$ & $0.285_{-0.020}^{+0.015}$ \\
Union2 & $(\mathrm{M})$ & $-0.97_{-0.12}^{+0.11}$ & $-1.23_{-0.32}^{+0.31}$ & $-0.79_{-0.17}^{+0.29}$ & $0.279_{-0.018}^{+0.013}$ \\
\hline Constitution mock & $(\mathrm{PP})$ & $-1.02_{-0.17}^{+0.17}$ & $-0.93_{-0.47}^{+0.43}$ & $-0.92_{-0.35}^{+0.30}$ & $0.277_{-0.017}^{+0.015}$ \\
Constitution mock & $(\mathrm{PM})$ & $-1.03_{-0.17}^{+0.17}$ & $-0.92_{-0.45}^{+0.46}$ & $-1.02_{-0.47}^{+0.28}$ & $0.278_{-0.014}^{+0.017}$ \\
Constitution mock & $(\mathrm{MM})$ & $-1.03_{-0.17}^{+0.17}$ & $-0.91_{-0.46}^{+0.45}$ & $-1.23_{-0.26}^{+0.49}$ & $0.278_{-0.017}^{+0.014}$ \\
\hline
\end{tabular}

while that for $w_{2}$ has a non-symmetric shape with a tail in the direction to which $w_{2}$ decreases. We expect from the $\mathrm{PP}$ value together with $\pm 1 \sigma$ errors listed in Table 1 that the $\mathrm{P}$ distribution for $w_{2}$ is also asymmetric and has a tail in the same direction and a peak location larger than that of the $\mathrm{M}$ distribution. In the increasing direction of $w_{2}$ the parameter is constrained by the big-bang nucleosynthesis calculation and the CMB shift parameter. On the other hand, $w_{2}$ is free to randomly walk in the opposite direction without a strong prior like $w_{2}>-5$ (see Sec. III) since the subdominant dark energy component at high redshift has more freedom to take any value of equation of state parameter. That is the reason why the likelihood distribution of $w_{2}$ parameter has a tendency of having the asymmetric shape with a tail to the smaller $w_{2}$ direction. Thus, it is naturally expected that the location of peak $(\mathrm{P})$ in the one-dimensional marginalized distribution is larger than the arithmetic mean (M) of MCMC chain elements.

We made similar analysis based on Union2 mock data sets, and the results are shown in Fig. 4 and Table [I] Here, we choose the transition redshifts $z_{\operatorname{tr} 1}=0.1$ and $z_{\mathrm{tr} 2}=0.3$, for which the deviations of $w_{i}$ 's from $w=-1$ are the largest in the case of Union2 data set (see Fig. 2). In the case of Union 2 set, $1 \sigma$ confidence limits of $w_{1}$ and $w_{2}$ deviate from $w=-1$ whereas $w_{0}$ is consistent with $\Lambda$ CDM. However, all the values of $w_{i}$ 's from the Union2, Union, Constitution, and Constitution- $U$ sets are within $2 \sigma$ limits thus are consistent with $\Lambda \mathrm{CDM}$ model (top row of Fig. 4). We also see that compared with the $M$ distributions for $\Lambda \mathrm{CDM}$ based Union2 mock data sets all $\mathrm{M}$ values of $w_{i}$ 's are consistent with $\Lambda \mathrm{CDM}$ model and deviations are not statistically significant. The $\mathrm{M}$ distributions of $w_{1}$ and $w_{2}$ (bottom row of Fig. 4) are narrower than those for Constitution mock data sets (bottom row of Fig. 3), reflecting that the dark energy equation of state parameters are relatively well constrained as we have more SNIa data points in the redshift bins.

We observe the same trend that $\mathrm{P}$ value is larger than $\mathrm{M}$ value for $w_{2}$ parameter, $w_{2}(\mathrm{P})>w_{2}(\mathrm{M})$. But the difference between $\mathrm{P}$ and $\mathrm{M}$ values is smaller due to the increase of the width of the final redshift bin $(z \geq 0.3)$ and of SNIa data points within the bin. As shown in Table II, the Union2 mock data analysis implies that estimated $w_{0}$ and $w_{1}$ are unbiased by three average methods (PP, PM, and MM); they are almost the same as the fiducial value $w=-1$. The PP, PM, and MM average methods for $w_{2}$ parameter give different values, indicating the likelihood distribution for this parameter is also not symmetric. However, the asymmetry is weaker than the case of Constitution data set.

\section{CHARACTERS OF CURRENT SNIA DATA BASED ON MOCK DATA ANALYSIS}

In the double-transition model the Constitution mock data sets shows some statistical deviation of equation of state parameters from the fiducial $\Lambda$ CDM model (Table II). For PM average, the value $w_{1}=-0.92$ indicates a bias at intermediate redshift interval although it is small compared with the size of $1 \sigma$ error. In the Constitution sample, the SNIa data points are irregularly distributed between $z=0.015$ and $z=1.551$ with non-uniform distance modulus errors, and the data is sparse in the intermediate redshift $z=0.1-0.3$. In this section, we investigate a bias that may be induced by the characters of the current SNIa data sets. The estimation of the bias is important because it can be misinterpreted as the spurious evolution of dark energy.

We have generated $\Lambda$ CDM based mock data sets in three different ways. First, we made SNIa data with uniform distribution in the redshift interval and uniform distance modulus errors (Case 1). We arrange SNIa 

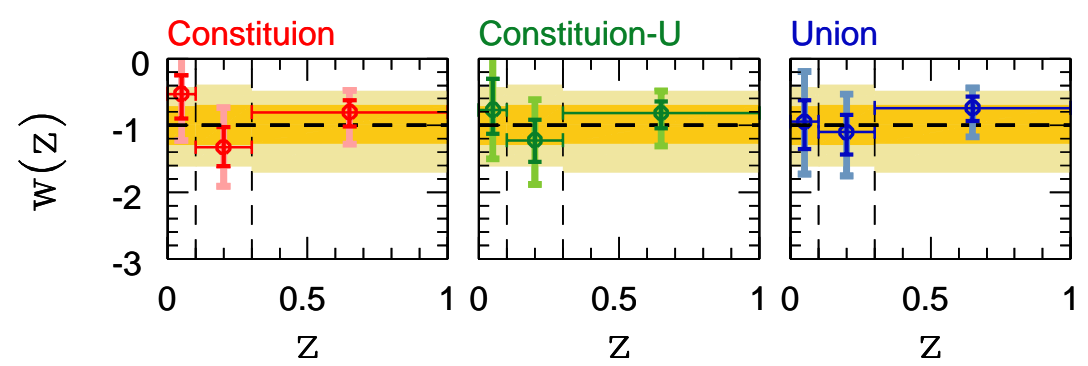

Union2
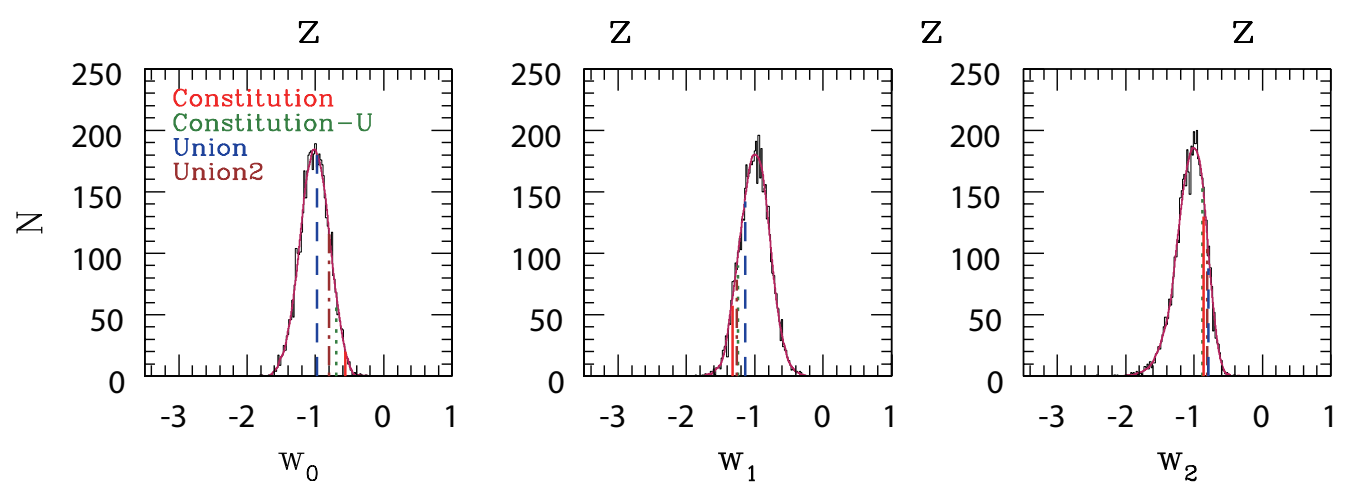

FIG. 4: The same as Fig. 3 but for $z_{\operatorname{tr} 1}=0.1$ and $z_{\operatorname{tr} 2}=0.3$ and based on Union2 mock data sets. The three bins in Union2 set have 165, 117, and 275 SNIa data points, respectively. In the top panels, data points with error bars are based on the actual SNIa data sets while the shaded regions (in all panels) are obtained from the five thousand $\Lambda$ CDM based Union2 mock data sets.

TABLE II: The same as Table \but for $z_{\operatorname{tr} 1}=0.1$ and $z_{\operatorname{tr} 2}=0.3$ and based on Union2 mock data sets.

\begin{tabular}{cccccc}
\hline Data & Average & $w_{0}$ & $w_{1}$ & $w_{2}$ & $\Omega_{\mathrm{m} 0}$ \\
\hline Constitution & $(\mathrm{P})$ & $-0.53_{-0.37}^{+0.28}$ & $-1.33_{-0.29}^{+0.30}$ & $-0.80_{-0.21}^{+0.18}$ & $0.285_{-0.017}^{+0.017}$ \\
Constitution-U & $(\mathrm{P})$ & $-0.77_{-0.36}^{+0.46}$ & $-1.22_{-0.32}^{+0.31}$ & $-0.82_{-0.22}^{+0.18}$ & $0.280_{-0.017}^{+0.020}$ \\
Union & $(\mathrm{P})$ & $-0.95_{-0.41}^{+0.32}$ & $-1.10_{-0.33}^{+0.25}$ & $-0.74_{-0.20}^{+0.17}$ & $0.280_{-0.017}^{+0.019}$ \\
Union2 & $(\mathrm{P})$ & $-0.80_{-0.20}^{+0.21}$ & $-1.26_{-0.21}^{+0.20}$ & $-0.77_{-0.18}^{+0.16}$ & $0.278_{-0.015}^{+0.015}$ \\
\hline Constitution & $(\mathrm{M})$ & $-0.56_{-0.34}^{+0.31}$ & $-1.32_{-0.30}^{+0.29}$ & $-0.86_{-0.15}^{+0.26}$ & $0.286_{-0.016}^{+0.018}$ \\
Constitution-U & $(\mathrm{M})$ & $-0.70_{-0.43}^{+0.39}$ & $-1.24_{-0.30}^{+0.33}$ & $-0.88_{-0.16}^{+0.24}$ & $0.283_{-0.014}^{+0.023}$ \\
Union & $(\mathrm{M})$ & $-0.98_{-0.38}^{+0.35}$ & $-1.14_{-0.29}^{+0.29}$ & $-0.79_{-0.15}^{+0.22}$ & $0.282_{-0.019}^{+0.017}$ \\
Union2 & $(\mathrm{M})$ & $-0.80_{-0.20}^{+0.21}$ & $-1.27_{-0.20}^{+0.21}$ & $-0.81_{-0.14}^{+0.20}$ & $0.280_{-0.017}^{+0.013}$ \\
\hline Union2 mock & $(\mathrm{PP})$ & $-1.00_{-0.21}^{+0.21}$ & $-1.00_{-0.22}^{+0.21}$ & $-0.95_{-0.22}^{+0.18}$ & $0.276_{-0.014}^{+0.015}$ \\
Union2 mock & $(\mathrm{PM})$ & $-1.00_{-0.22}^{+0.21}$ & $-1.00_{-0.22}^{+0.22}$ & $-1.01_{-0.22}^{+0.20}$ & $0.278_{-0.016}^{+0.015}$ \\
Union2 mock & $(\mathrm{MM})$ & $-1.01_{-0.21}^{+0.20}$ & $-1.00_{-0.22}^{+0.22}$ & $-1.06_{-0.17}^{+0.25}$ & $0.278_{-0.016}^{+0.015}$ \\
\hline
\end{tabular}

data points $\left(N_{\mathrm{SN}}=397\right)$ uniformly on the redshift range spanned by the Constitution sample and assign the uniform error to all members. We take the harmonic average as the uniform error given by

$$
\bar{\sigma}=\sqrt{\frac{N_{\mathrm{SN}}}{\sum_{i=1}^{N_{\mathrm{SN}}} 1 / \sigma_{\mathrm{obs}, i}^{2}}}=0.177,
$$

in magnitude scale. The second-type mock data sets have the uniform redshift-distribution but with distance modulus errors of the Constitution sample (Case 2). We sort the Constitution SNIa data points in increasing order in redshift, then assign distance modulus errors to mock SNIa members in the same order. The third-type mock data sets have the same redshift-distribution as the Constitution data set but with uniform distance modulus errors (Case 3). For comparison, we include the Constitution mock data sets as Case 4; these are already presented in Table I. We have analyzed these mock data sets (including mock BAO and $\mathrm{CMB}$ parameters) in the same way as the Constitution mock data sets have been analyzed for double-transition model with $z_{\operatorname{tr} 1}=0.2$ and $z_{\operatorname{tr} 2}=0.4$ (Sec. IV). The results are shown in Table III. which compares dark energy equation of state parameters 
obtained by using different average estimation methods (PP, PM, and MM).

For all Cases $1-4$, we see that $w_{0}$ and $w_{1}$ almost do not depend on the choice of average methods, that is, $w_{i}(\mathrm{PP}) \simeq w_{i}(\mathrm{PM}) \simeq w_{i}(\mathrm{MM})$, while $w_{2}$ parameters show a trend shown in the previous section, $w_{2}(\mathrm{PP})>w_{2}(\mathrm{PM})>w_{2}(\mathrm{MM})$. The likelihood distributions of $w_{0}$ and $w_{1}$ are symmetric whereas that of $w_{2}$ is not symmetric for all cases of mock data sets. This is verified in Fig. 5 which compares distributions of $\mathrm{M}$ values derived from the four cases of $\Lambda$ CDM mock data sets. Expecting that analysis of Case 1 (uniform distribution and error) restores the fiducial value $w=-1$ correctly, we found that the PM acts as the unbiased (or less biased) estimator for the average of parameter because PM values of $w_{0}, w_{1}$, and $w_{2}$ are consistent with the $\Lambda$ CDM model. Even for the non-symmetric shape of likelihood distribution as in the case of $w_{2}$ parameter, the PM average is unbiased, and thus provides the precise estimation of bias in the parameter.

For Constitution mock data sets (Case 4), the bias in the positive direction for $w_{1}$ parameter is observed. Because the mock data sets of Case 3 (uniform error) induce the similar amount of bias in the same direction while the data sets of Case 2 (uniform distribution) does not affect $w_{1}$ much, we conclude that the irregular distribution of Constitution SNIa data points is more concerned with the bias than the non-uniform distance modulus errors. However, the bias estimated based on the $\Lambda \mathrm{CDM}$ mock data sets is small compared with the precision the current SNIa data (including BAO and CMB parameters) allows. For Constitution mock data (Case 4), it amounts to $1+w_{1} \simeq 0.18 \sigma$ at most for PM average. Unlike the bias in the $w_{1}$ parameter, $w_{2}$ is not much biased (based on PM average).

For the Union2 mock data sets, we have not seen any significant deviation of equation of state parameters (see Table III). Because of the relatively denser SNIa data points, the biases in $w_{i}$ estimation are smaller than those of Constitution case. However, the likelihood distribution of $w_{2}$ still appears asymmetric (as shown in Fig. 4), and thus the estimated value of $w_{2}$ depends on the average methods.

\section{DISCUSSION}

In this study, we have investigated the evolution of dark energy equation of state parameter $w(z)$ using the sudden-jump approximation of $w$ for some chosen redshift intervals with double transitions. We used four SNIa data (Constitution, Constitution-U, Union, and Union2) together with $\mathrm{BAO} A$ parameter and CMB $R$ parameter, and used the MCMC method with Metropolis-Hastings algorithm to obtain the likelihood in the parameter space. In order to test the statistical significance of, or to estimate the bias in, the equation of state parameters we investigated the case of $\Lambda \mathrm{CDM}$ mock data sets. Since the analysis of thousands of $\Lambda \mathrm{CDM}$ mock data sets can include all statistical effects due to the characters of data sets such as the irregular distribution in the redshift inter$\mathrm{val}$ and the different random realization of measurement errors, our method of using the $\Lambda$ CDM mock data is complementary to the uncorrelated estimation of dark energy equation of state parameters based on the decorrelating technique [13, 22, 28].

In the double-transition dark energy models, we show that there are some deviations from $\Lambda$ CDM in the low $(z \lesssim 0.2)$ and the middle $(0.2 \lesssim z \lesssim 0.4)$ redshift regions especially in the Constitution and Constitution- $\mathrm{U}$ sets. Deviations of $w_{i}$ 's from $\Lambda \mathrm{CDM}$ in the low and the middle redshift are between 1 and $2 \sigma$ confidence levels, see the top row of Fig. 3. The deviations are stronger when we compare each arithmetic mean $(\mathrm{M})$ value of $w_{i}$ with the $\Lambda \mathrm{CDM}$ mock data; the $\mathrm{M}$ values of Constitution and Constitution- $\mathrm{U}$ sets are situated near $2 \sigma$ boundary of the $\Lambda$ CDM mock data distribution, see the bottom row of Fig. 3, whereas there are no such deviations in the Union set. Thus, we conclude that our analysis of the double-transition model indicates statistically noticeable deviation of the Constitution data from $\Lambda$ CDM model in the low redshift. Furthermore, consistent result between Constitution set and Constitution- $U$ set suggests that the detected deviation in the Constitution data is not due to additional data in the Constitution data, but mainly due to different calibration of the same Union data. Results of Constitution-U compared with the Constitution and the Union data in Figs. 2 and 3 apparently show that the new 90 CfA3 SNIa data added in the Constitution data do not have role in causing the difference between the Constitution and the Union sets.

Our conclusion is still valid when we measure dark energy equation of state parameters by choosing different transition redshifts, as summarized in Fig. 6] (top three rows), where the width of the second redshift bin has been extended by the amount of $\Delta z=0.1$ to left, right, and both directions. Although extending the redshift interval of the middle redshift bin makes the error bar of $w_{1}$ value smaller as expected, the observed trend for $w_{0}$ and $w_{1}$ estimates of Constitution, Constitution- $\mathrm{U}$, and Union samples does not change much in comparison with the result shown in Fig. 3 .

In the bottom row of Fig. 6 we present our measurement of equation of state parameters based on the tripletransition model with the first three redshift bins equally spaced between $z=0$ and 0.4. Even in this case our conclusion maintains. However, increasing number of redshift bins causes error bars to be drastically larger and it is more and more difficult to make the MCMC chains converge without strong priors. This is why we have chosen a simple dark energy model with double transitions.

We emphasize that the Union and the Constitution$\mathrm{U}$ sets are composed of the same SNIa members and are based on the same light-curve fit parameters. In a dark energy model with constant equation of state, the $w$ parameter ( $\mathrm{P}$ value) estimated from SNIa data together 

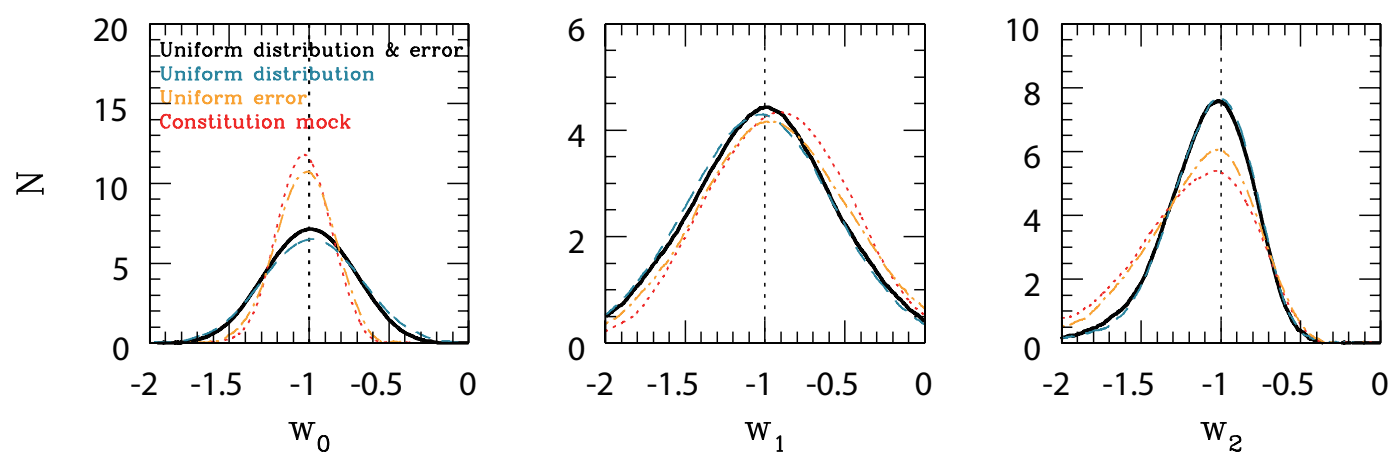

FIG. 5: Distributions of $\mathrm{M}$ values for four different types of $\Lambda$ CDM mock data sets. The smooth distributions have been made based on the histograms with the bin size $\Delta w=0.001$. Black (solid), blue (dashed), yellow (dot-dashed), and red (dotted) curves represent Case 1 (uniform distribution \& error), Case 2 (uniform distribution), Case 3 (uniform error), and Case 4 (Constitution mock), respectively. Vertical dashed lines indicate the location of $w=-1$.

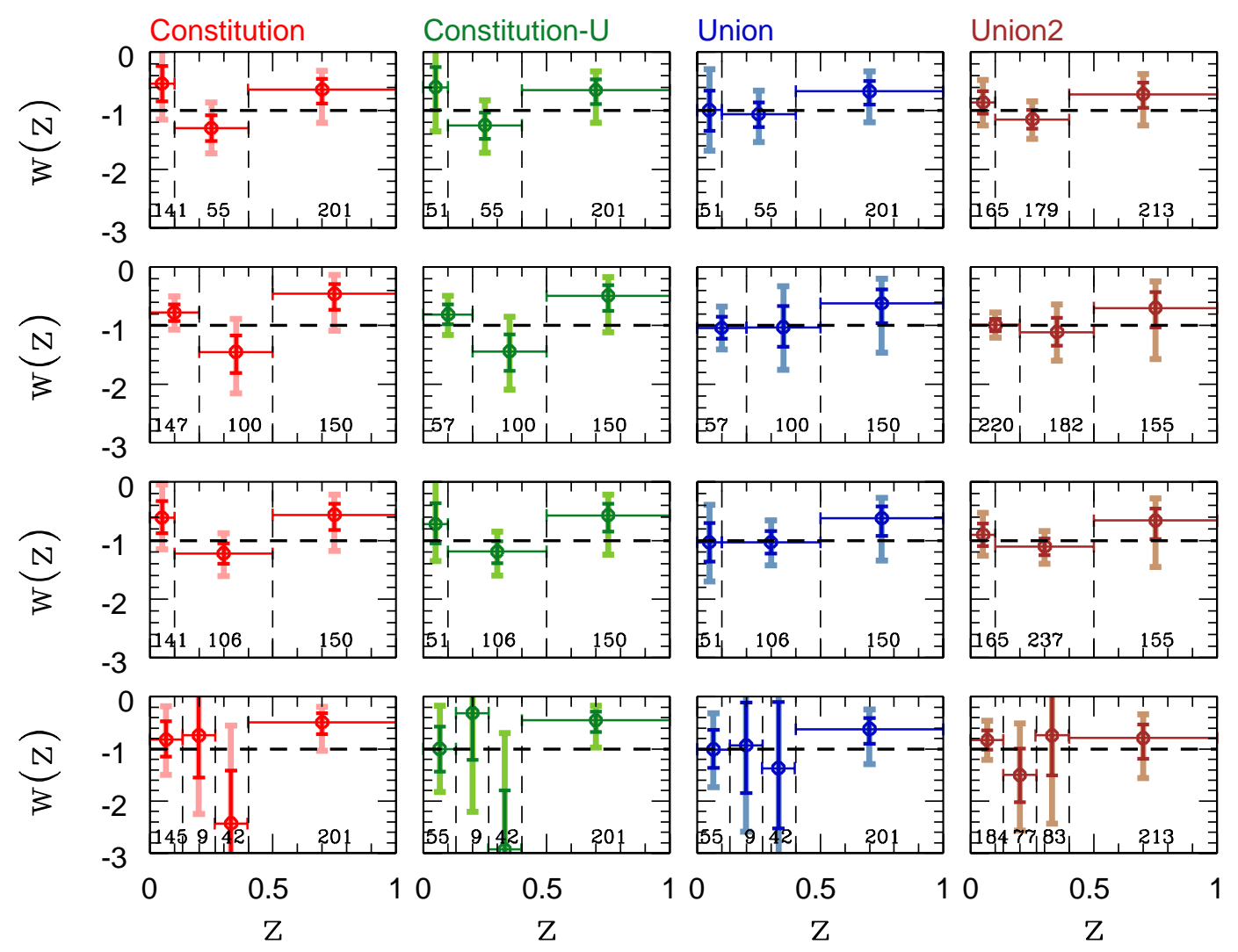

FIG. 6: Top three rows: dark energy equation of state parameters $\left(w_{0}, w_{1}, w_{2}\right)$ measured from Constitution, Constitution-U, Union, and Union2 samples for different choices of transition redshifts, $z_{\operatorname{tr} 1}$ and $z_{\operatorname{tr} 2}$. In order from top to bottom we choose $(a)$ $z_{\operatorname{tr} 1}=0.1$ and $z_{\operatorname{tr} 2}=0.4,(b) z_{\operatorname{tr} 1}=0.2$ and $z_{\operatorname{tr} 2}=0.5$, and $(c) z_{\operatorname{tr} 1}=0.1$ and $z_{\operatorname{tr} 2}=0.5$. Bottom row: $(d)$ dark energy equation of state parameters in the triple-transition dark energy model. The first three bins are equally spaced in the redshift interval $z=0-0.4$. Each number listed in each redshift bin indicates the number of SNIa included in the bin. Note that although the size of error bars generally gets smaller as the corresponding redshift bin includes more SNIa data points, it is also affected by the quality of SNIa data points within the bin. 
TABLE III: Dark energy equation of state parameters of double-transition model with $z_{\operatorname{tr} 1}=0.2$ and $z_{\operatorname{tr} 2}=0.4$ estimated from the five thousand $\Lambda$ CDM based SNIa mock data sets with four different types (Cases 1-4; see text). Values are listed based on three average estimation methods (PP, PM, and MM). The errors indicate $68.3 \%$ confidence levels.

\begin{tabular}{lrrr}
\hline Data & $w_{0}(\mathrm{PP})$ & $w_{1}(\mathrm{PP})$ & $w_{2}(\mathrm{PP})$ \\
\hline Case 1: uniform distribution and error & $-0.99_{-0.27}^{+0.27}$ & $-1.00_{-0.47}^{+0.45}$ & $-0.96_{-0.25}^{+0.22}$ \\
Case 2: uniform distribution & $-0.97_{-0.30}^{+0.29}$ & $-1.03_{-0.47}^{+0.46}$ & $-0.95_{-0.25}^{+0.22}$ \\
Case 3: uniform error & $-1.01_{-0.19}^{+0.18}$ & $-0.97_{-0.50}^{+0.46}$ & $-0.92_{-0.34}^{+0.27}$ \\
Case 4: Constitution mock & $-1.02_{-0.17}^{+0.17}$ & $-0.93_{-0.47}^{+0.43}$ & $-0.92_{-0.35}^{+0.30}$ \\
\hline Data & $w_{0}(\mathrm{PM})$ & $w_{1}(\mathrm{PM})$ & $w_{2}(\mathrm{PM})$ \\
\hline Case 1: uniform distribution and error & $-1.00_{-0.28}^{+0.28}$ & $-1.00_{-0.47}^{+0.48}$ & $-1.01_{-0.29}^{+0.24}$ \\
Case 2: uniform distribution & $-0.98_{-0.31}^{+0.30}$ & $-1.01_{-0.49}^{+0.45}$ & $-1.03_{-0.27}^{+0.25}$ \\
Case 3: uniform error & $-1.01_{-0.19}^{+0.17}$ & $-0.95_{-0.49}^{+0.48}$ & $-1.02_{-0.27}^{+0.24}$ \\
Case 4: Constitution mock & $-1.03_{-0.17}^{+0.17}$ & $-0.92_{-0.45}^{+0.46}$ & $-1.02_{-0.47}^{+0.28}$ \\
\hline Data & $w_{0}(\mathrm{MM})$ & $w_{1}(\mathrm{MM})$ & $w_{2}(\mathrm{MM})$ \\
\hline Case 1: uniform distribution and error & $-1.00_{-0.28}^{+0.28}$ & $-1.01_{-0.46}^{+0.49}$ & $-1.10_{-0.20}^{+0.33}$ \\
Case 2: uniform distribution & $-0.98_{-0.31}^{+0.30}$ & $-1.04_{-0.46}^{+0.48}$ & $-1.08_{-0.22}^{+0.30}$ \\
Case 3: uniform error & $-1.03_{-0.17}^{+0.19}$ & $-0.94_{-0.48}^{+0.47}$ & $-1.17_{-0.12}^{+0.39}$ \\
Case 4: Constitution mock & $-1.03_{-0.17}^{+0.17}$ & $-0.91_{-0.46}^{+0.45}$ & $-1.23_{-0.26}^{+0.07}$ \\
\hline
\end{tabular}

with BAO $A$ parameter is $1+w=-0.011_{-0.080}^{+0.074}$ for Union sample and $1+w=-0.010_{-0.080}^{+0.078}$ for Constitution- $U$ sample, both of which are nearly identical to each other and are similar to the values obtained by Refs. 7, 8]. Although the two data sets give nearly identical results in the simple constant $w$ dark energy model, they show different behaviors of dark energy equation of state in our evolving dark energy model with double transitions, due to different calibrations applied during the production of SNIa distance modulus. Our analysis suggests that it is generally important to distinguish the real $w$ evolution from the calibration artefact in the SNIa data analysis.

However, we cannot conclude that such deviations in the Constitution data are the strong hint for evolution of dark energy because all the equation of state parameters $w_{i}$ 's measured from all the observational data sets are consistent with $\Lambda$ CDM model within $2 \sigma$ confidence limits. Our results are also consistent with previous studies searching for dark energy evolution with SNIa data based on the redshift-binning method [9, 33 39]. Furthermore, the difference between Union and Constitution- $U$ due to different calibrations is also not significant at the level of current precision of SNIa data. Such an effect caused by different calibrations should be considered to be important in the forthcoming missions of SNIa survey.

From the analysis of $\Lambda$ CDM-motivated mock data sets with four different types (Cases 1-4), we have tried to estimate bias in the equation of state parameters due to the character of data set used in search for dark energy evolution. Comparing average values for $w_{i}$ 's obtained by three different methods such as PP (peak of peaks),
PM (peak of means), and MM (mean of means) while knowing the answer $(w=-1)$, we conclude that the PM is an unbiased estimator of average bias. For the symmetric likelihood distribution, all average methods give values consistent with each other (e.g., see Fig. 3 and $w_{0}$ and $w_{1}$ for the Constitution data sets in Table III). However, for the non-symmetric likelihood distribution as in the case of $w_{2}$ parameter, the PP and MM averages are biased to the opposite directions with respect to the PM average which restores the fiducial value $w=-1$ correctly. The Constitution sample turns out to induce a bias in the $w_{1}$ parameter by the amount of about $0.2 \sigma$ whereas the Union2 sample does not show any noticeable bias in the estimation of parameters (see Table III). In order to precisely estimate any bias due to the character of data set based on the mock data analysis, we suggest to use the PM quantity, the peak (or mode) of the distribution of arithmetic means, as the unbiased estimator for the average bias.

\section{Acknowledgments}

We wish to thank Dr. Eric V. Linder, Dr. Yong-Seon Song, and Professor Dal Ho Kim for useful comments and discussions. We also would like to thank the anonymous referee for the constructive and helpful comments on our manuscript. This work was supported by the Korea Research Foundation Grant funded by the Korean Government (KRF-2008-341-C00022). 
[1] A.G. Riess et al. Astron. J. 116, 1009 (1998) arXiv:astro-ph/9805201.

[2] S. Perlmutter et al. Astrophys. J. 517, 565 (1999) arXiv:astro-ph/9812133.

[3] A.G. Riess et al. Astrophys. J. 659, 98 (2007) arXiv:astro-ph/0611572.

[4] P. Astier et al. Astron. Astrophys. 447, 31 (2006) arXiv:astro-ph/0510447.

[5] T.M. Davis et al. Astrophys. J. 666, 716 (2007) arXiv:astro-ph/0701510.

[6] H. Lampeitl et al. Mon. Not. R. Astron. Soc. 401, 2331 (2009) arXiv:0910.2193 [astro-ph.CO]].

[7] Supernova Cosmology Project Collaboration, M. Kowalski et al. Astrophys. J. 686, 749 (2008) arXiv:0804.4142 [astro-ph]].

[8] M. Hicken et al. Astrophys. J. 700, 1097 (2009) arXiv:0901.4804 [astro-ph.CO]].

[9] R. Amanullah, et al. Astrophys. J. 716, 712 (2010) arXiv:1004.1711 [astro-ph.CO]].

[10] G. Efstathiou, Mon. Not. R. Astron. Soc. 301, 842 (1999) arXiv:astro-ph/9904356.

[11] D. Huterer and M.S. Turner, Phys. Rev. D 64, 123527 (2001) arXiv:astro-ph/0012510.

[12] J. Weller and A. Albrecht, Phys. Rev. Lett. 86, 1939 (2001) arXiv:astro-ph/0008314.

[13] D. Huterer and G. Starkman, Phys. Rev. Lett. 90, 031301 (2003) arXiv:astro-ph/0207517.

[14] P.S. Corasaniti and E.J. Copeland, Phys. Rev. D 67, 063521 (2003) arXiv:astro-ph/0205544.

[15] U. Alam, V. Sahni, T.D. Saini and A.A. Starobinsky, Mon. Not. R. Astron. Soc. 354, 275 (2004) arXiv:astro-ph/0311364.

[16] R.A. Daly and S.G. Djorgovski, Astrophys. J. 597, 9 (2003) arXiv:astro-ph/0305197.

[17] A.G. Kim, E.V. Linder, R. Miquel and N. Mostek, Mon. Not. R. Astron. Soc. 347, 909 (2004) arXiv:astro-ph/0304509.

[18] H.K. Jassal, J.S. Bagla and T. Padmanabhan, Mon. Not. R. Astron. Soc. 356, L11 (2005) arXiv:astro-ph/0404378.

[19] R.A. Daly and S.G. Djorgovski, Astrophys. J. 612, 652 (2004) arXiv:astro-ph/0403664.

[20] C. Wetterich, Phys. Lett. B 594, 17 (2004) arXiv:astro-ph/0403289.

[21] Y.G. Gong, Class. Quant. Grav. 22, 2121 (2005) arXiv:astro-ph/0405446.

[22] D. Huterer and A. Cooray, Phys. Rev. D 71, 023506 (2005) arXiv:astro-ph/0404062.

[23] E.V. Linder and D. Huterer, Phys. Rev. D 72, 043509 (2005) arXiv:astro-ph/0505330.

[24] Y.G. Gong and Y.Z. Zhang, Phys. Rev. D 72, 043518 (2005) arXiv:astro-ph/0502262.

[25] A. Shafieloo, U. Alam, V. Sahni and A.A. Starobinsky, Mon. Not. R. Astron. Soc. 366, 1081 (2006) arXiv:astro-ph/0505329.

[26] A. Shafieloo, Mon. Not. R. Astron. Soc. 380, 1573 (2007) arXiv:astro-ph/0703034.

[27] R. de Putter and E.V. Linder, Astropart. Phys. 29, 424 (2008) arXiv:0710.0373 [astro-ph]].
[28] S. Sullivan, A. Cooray and D.E. Holz, JCAP 0709, 004 (2007) arXiv:0706.3730 [astro-ph]].

[29] A. Hojjati, L. Pogosian and G.B. Zhao, JCAP 1004, 007 (2010) arXiv:0912.4843 [astro-ph.CO]].

[30] J.B. Jimenez, R. Lazkoz and A.L. Maroto, Phys. Rev. D 80, 023004 (2009) arXiv:0904.0433 2 [astro-ph.CO]].

[31] Y. Wang, Phys. Rev. D 80, 123525 (2009) arXiv:0910.2492 [astro-ph.CO]].

[32] A. Shafieloo, V. Sahni and A.A. Starobinsky, Phys. Rev. D 80, 101301 (2009) arXiv:0903.5141 [astro-ph.CO]].

[33] Q.G. Huang, M. Li, X.D. Li and S. Wang, Phys. Rev. D 80, 083515 (2009) arXiv:0905.0797 [astro-ph.CO]].

[34] R.G. Cai, Q. Su and H.B. Zhang, JCAP 1004, 012 (2010) arXiv:1001.2207 [astro-ph.CO]].

[35] G.B. Zhao and X. Zhang, Phys. Rev. D 81, 043518 (2010) arXiv:0908.1568 [astro-ph.CO]].

[36] Y.G. Gong, R.G. Cai, Y. Chen and Z.H. Zhu, JCAP 1001, 019 (2010) arXiv:0909.0596 [astro-ph.CO]].

[37] P. Serra, A. Cooray, D.E. Holz, A. Melchiorri, S. Pandolfi and D. Sarkar, Phys. Rev. D 80, 121302 (2009) arXiv:0908.3186 [astro-ph.CO]].

[38] S. Wang, X.-D. Li, and M. Li, arXiv:1009.5837 v3 [astroph.CO].

[39] S. Qi, T. Lu and F.-Y. Wang, Mon. Not. R. Astron. Soc. 398, 78 (2009) arXiv:0904.2832 [astro-ph.CO].

[40] M. Chevallier and D. Polarski, Int. J. Mod. Phys. D 10, 213 (2001) arXiv:gr-qc/0009008.

[41] E.V. Linder, Phys. Rev. Lett. 90, 091301 (2003) arXiv:astro-ph/0208512.

[42] D.J. Eisenstein et al. [SDSS Collaboration], Astrophys. J. 633, 560 (2005) arXiv:astro-ph/0501171.

[43] E. Komatsu et al. [WMAP Collaboration], Astrophys. J. Suppl. 180, 330 (2009) arXiv:0803.0547 [astro-ph]].

[44] J.R. Bond, G. Efstathiou and M. Tegmark, Mon. Not. R. Astron. Soc. 291, L33 (1997) arXiv:astro-ph/9702100.

[45] Y. Wang and P. Mukherjee, Astrophys. J. 650, 1 (2006) arXiv:astro-ph/0604051.

[46] N. Metropolis, A.W. Rosenbluth, M.N. Rosenbluth, A.H. Teller and E. Teller, J. Chem. Phys. 21, 1087 (1953).

[47] W.K. Hastings, Biometrika 57, 97 (1970).

[48] R. Bean, S.H. Hansen, and A. Melchiorri, Phys. Rev. D 64, 103508 (2001) arXiv:astro-ph/0104162 ; B.A. Bassett et al., JCAP 07, 007 (2008) arXiv:0709.0526].

[49] A. Abrahamse, A. Albrecht, M. Barnard, and B. Bozek, Phys. Rev. D 77, 103503 (2008) arXiv:0712.2879 [astro$\mathrm{ph}]$.

[50] T. Holsclaw, U. Alam, B. Sanso, H. Lee, K. Heitmann, S. Habib and D. Higdon, Phys. Rev. D 82, 103502 (2010) arXiv:1009.5443 [astro-ph.CO].

[51] I. Antoniou and L. Perivolaropoulos, arXiv:1007.4347 [astro-ph.CO].

[52] D.N. Spergel, et al., Astrophys. J. Suppl. 148, 175 (2003) arXiv:astro-ph/0302209.

[53] W.H. Press, S.A. Teukolsky, W.T. Vetterling, and B.P. Flannery, Numerical Recipes in Fortran. The art of scientific computing, 2nd eds. (Cambridge University Press, 1992), p. 644 . 